\title{
Factores motivacionales y experiencia autotélica en el ejercicio físico: propuesta de un modelo explicativo*
}

\author{
Motivational Factors and Autotelic Experience \\ in Physical Exercise: an Explanatory Model
}

\begin{tabular}{l|l|l} 
Recibido: marzo 2 de 2010 | Revisado: julio 5 de 2010 Aceptado: agosto 14 de 2010
\end{tabular}

\author{
Álvaro SicIlia CAMACHO ** \\ CORNelio ÁGuila Soto \\ DAVID GONZÁLEZ-CUTRE ${ }^{* * * *}$ \\ Universidad de Almería, España \\ JuAN ANTONIO MORENO-MurCiA ${ }^{* * *}$ \\ Universidad Miguel Hernández, Elche, España
}

Para citar este artículo. Sicilia, A., Águila, C., González-Cutre, D., \& Moreno-Murcia, J. A. (2011). Factores motivacionales y experiencia autotélica en el ejercicio físico: propuesta de un modelo explicativo. Universitas Psychologica, 10(1), 125-135.

Artículo de investigación. Investigación financiada por el Ministerio de Ciencia e Innovación de España, a través del proyecto de investigación "Factores motivacionales relacionados con la adherencia a la práctica física: análisis en contextos de actividad física no competitiva" (Ref. DEP2007-73201-CO3-03/ ACTI).

** Facultad de Ciencias de la Educación. Universidad de Almería. Grupo de Investigación Hum-628. Ctra. de Sacramento s/n 04120. La Cañada de San Urbano, Almería, España. Tel: 950-015366. Fax: 950015839.E-mails: asicilia@ual.es; Cornelio@ual.es.

***** Universidad Miguel Hernández de Elche. Centro de Investigación del Deporte, Avda. de la Universidad, s/n. 03202 Elche, Alicante, España. Tel.: 965-222162.E-mail:j.moreno@umh.es, dgonzalezcutre@umh.es
RESUMEN

El objetivo de este estudio fue analizar la influencia estimada del clima motivacional percibido, las creencias implícitas de habilidad y la motivación intrínseca, sobre la propensión a la experiencia autotélica en el ejercicio físico. 727 practicantes (402 hombres y 325 mujeres), con edades comprendidas entre los 16 y 78, respondieron a diferentes cuestionarios. Los resultados del modelo de ecuaciones estructurales revelaron que el clima-tarea predecía positivamente la creencia incremental, la motivación intrínseca y la experiencia autotélica, mientras que el clima-ego predecía positivamente la creencia de entidad. La creencia incremental predecía positivamente la motivación intrínseca, mientras que la creencia de entidad la predecía negativamente. La motivación intrínseca predecía positivamente la experiencia autotélica. El modelo fue invariante por sexo. Los resultados son discutidos de acuerdo a la importancia que puede jugar el personal de los centros deportivos para promover una práctica física continuada.

Palabras clave autores

Motivación, creencias de habilidad, experiencia autotélica, actividad física.

Palabras clave descriptores

Actividad motora, motivación, fisiología, ejercicio.

\section{A B S T R A C T}

The purpose of this study was to analyse the estimated influence of perception of the motivational climate, implicit beliefs of ability and intrinsic motivation on the autotelic experience propensity in physical exercise. A sample of 727 practitioners of non-competitive physical-sport activities (402 men y 325 women), age between 16 and 78 years, participated in this study. The results of the structural equation model showed that the task climate positively predicted incremental belief, intrinsic motivation and autotelic experience, whereas the ego climate positively predicted entity belief. Incremental belief positively predicted intrinsic motivation, whereas entity belief negatively predicted the latter. Intrinsic motivation positively predicted autotelic experience. The model was invariant across gender. The results are discussed with reference to the important role staff could play in encouraging practitioners to continue to do sport.

Key words authors

Motivation, Implicit Beliefs of Ability, Autotelic Experience, Physical Activity.

Key words plus

Motor Activity, Motivation, Physiology, Exercise. 


\section{Introducción}

Hoy día se considera que la participación en actividades físicodeportivas proporciona notables beneficios para la salud física, psicológica y social (American College of Sport Medicine [ACSM], 1995). No obstante, conocer los beneficios de la actividad física regular no siempre es motivo suficiente para incorporarla al estilo de vida (Heyward, 2002; Mutrie \& Woods, 2003).

La teoría de la autodeterminación (Deci \& Ryan, 1985, 2000) está siendo una de las teorías psicológicas más utilizadas, para el estudio de la motivación en la práctica física. Esta teoría defiende la idea de que las conductas humanas pueden ser más o menos autodeterminadas en función del tipo de motivación que las sustenta. De este modo, la teoría plantea un continuo que iría desde la desmotivación hasta la motivación intrínseca, pasando por diferentes niveles de motivación extrínseca. Así, cuando una persona realiza una actividad por el placer y el disfrute que le produce, sin otros fines ni condicionantes externos, presentaría una conducta autodeterminada. Estudios en el campo de la actividad física y el deporte han mostrado que altos niveles de motivación intrínseca hacia el ejercicio físico, están relacionados positivamente con una mayor asistencia a las sesiones, una mayor adherencia (Ryan, Frederick, Lepes, Rubio \& Sheldon, 1997), así como un menor índice de abandono (Sarrazin, Vallerand, Guillet, Pelletier \& Cury, 2002).

Vallerand (2007) defiende la posición de que la motivación intrínseca favorece la aparición de patrones adaptativos-conductuales, cognitivos y afectivos, hacia el ejercicio, tales como el placer, el interés, el esfuerzo mantenido o la intención de seguir realizando actividad física. Una clara consecuencia positiva de la motivación intrínseca en el campo físicodeportivo es el llamado estado de flow (Jackson \& Csikszentmihalyi, 1999; Kowal \& Fortier, 1999).

Acuñado por Csikszentmilhalyi (1990, 1997), el término flow refiere a un estado de la mente de máxima concentración, en una tarea que resulta desafiante y que produce altas dosis de placer.
Aunque el flow ha sido identificado en tareas muy diferentes, la actividad físicodeportiva se muestra como una de las mejores fuentes para experimentarlo (Jackson \& Csikszentmilhalyi, 1999). Dentro de este campo, la mayoría de las investigaciones sobre flow se han centrado en el deporte competitivo (e.g., Jackson, 1996; Kowal \& Fortier, 2000), aunque existen estudios que muestran que puede darse en actividades recreativas (Jackson, Kimiecik, Ford \& Marsh, 1998; Stein, Kimiecik, Daniels \& Jackson, 1995).

Csikszentmilhalyi $(1990,1997)$ considera que el estado de flow tiene nueve factores, si bien la experiencia autotélica ha sido destacada como un de los factores que más fuertemente definiría la experiencia de flow. De hecho, algunos autores han resaltado que la experiencia autotélica sería el elemento más importante en la actividad física recreativa (Jackson, 1996; Jackson \& Marsh, 1996). La experiencia autotélica resaltaría precisamente la práctica realizada como una vivencia con finalidad en sí misma y, por tanto, intrínsecamente recompensada. Una tarea reconfortante que produce altas dosis de placer y que, por tanto, fomenta la adherencia y el compromiso hacia la actividad física regular (Jackson, 1996; Kimiecik, 2000).

Los estudios que han analizado las relaciones entre motivación y estado de flow en la práctica físicodeportiva muestran que la motivación intrínseca favorece la aparición de dicho estado (e.g., Jackson et al., 1998; Kowal \& Fortier, 1999, 2000). No obstante, no existen trabajos que hayan testado la relación de la experiencia autotélica y la motivación, en el ámbito de la actividad física recreativa.

La tendencia a percibir experiencias autotélicas, de acuerdo con Csikszentmilhalyi (1990), no sólo dependerá de condiciones innatas, sino también ambientales. De acuerdo al modelo de Vallerand $(1997,2001)$, debe asumirse la coexistencia de distintos factores antecedentes y mediadores afectando la percepción de experiencias autotélica. Parece interesante analizar qué condiciones se mostrarían favorables para el fomento de la motivación intrínseca y, a partir de ésta, para el desarrollo de experiencias autotélicas. Hasta la fecha, la investigación en actividad físicodeportiva ha 
mostrado que el clima motivacional percibido por los participantes y sus creencias sobre la habilidad en el deporte, pueden tener influencias sobre la motivación (e.g., Biddle, Wang, Chatzisarantis \& Spray, 2003; Ntoumanis \& Biddle, 1999; Wang \& Biddle, 2001).

De acuerdo con la perspectiva de la teoría de las metas de logro (Nicholls, 1989), hay dos tipos de climas motivacionales predominantes: un climatarea, donde se promociona la mejora personal, el esfuerzo y el aprendizaje; y un clima-ego, en el que se estimula la comparación y la rivalidad entre los miembros del grupo. Los estudios realizados en el ámbito de la actividad física y el deporte apuntan que el clima motivacional que implica a la tarea se encuentra relacionado positivamente con la motivación intrínseca, mientras que el clima motivacional que implica al ego no se relaciona o se relaciona negativamente con esta variable y otras consecuencias adaptativas al ejercicio (véase Ntoumanis \& Biddle, 1999).

El clima motivacional creado por los otros puede modular la adopción de un concepto de habilidad basado en la maestría o en el rendimiento en los diferentes contextos de ejecución (Nicholls, 1989). Las creencias implícitas de la habilidad se basan en las diferencias entre capacidad y esfuerzo, y cómo esta relación es entendida por las personas (Nicholls, 1992). De acuerdo con Biddle et al. (2003), existen dos tipos de creencias implícitas de habilidad deportiva: una creencia incremental, según la cual la habilidad puede ser mejorada a través del esfuerzo y el aprendizaje; y una creencia de entidad, que considera la habilidad como algo estable y, por tanto, que no es susceptible de modificarse a través del entrenamiento. Las relaciones entre los climas motivacionales y las creencias implícitas de habilidad no han sido muy estudiadas en entornos de actividad física recreativa. No obstante, Ommundsen (2001) encontró en clases de educación física que la percepción de un entorno de aprendizaje que prima la competición y la comparación social, predecía positivamente la creencia de entidad, mientras que la percepción de un clima que refuerza el esfuerzo, el progreso y el apoyo del profesor a todos los alumnos, predecía positivamente una creencia incremental.

Finalmente, las creencias implícitas sobre la habilidad en la práctica físicodeportiva han sido relacionadas con la motivación. La creencia de habilidad incremental se ha asociado positivamente con la motivación intrínseca, mientras que la creencia de entidad se ha relacionado negativamente con la motivación intrínseca y positivamente con la desmotivación ( $\mathrm{Li}$, Lee \& Solmon, 2005; Wang \& Biddle, 2001, 2003). Estos estudios sugieren que si una persona cree que su habilidad puede mejorar, es probable que disfrute más con el ejercicio físico, ya que sabe que si se esfuerza puede conseguir efectos positivos.

El objetivo de este trabajo fue testar un modelo explicativo de la experiencia autotélica en el ejercicio físico, tratando de integrar los principales modelos y teorías contemporáneas motivacionales. Se analizaron las relaciones entre los climas motivacionales percibidos por los participantes, sus creencias de habilidad y la motivación intrínseca, como predictores de la experiencia autotélica. Se hipotetizó que la percepción de un clima que implica a la tarea prediría positivamente la creencia incremental de habilidad, mientras que la percepción de un clima-ego prediría positivamente la creencia de entidad. La creencia incremental prediría positivamente la motivación intrínseca, mientras que la creencia de entidad lo haría de forma negativa. La motivación intrínseca prediría positivamente la propensión a la experiencia autotélica.

\section{Método}

\section{Participantes}

La muestra de este estudio estuvo compuesta por 727 practicantes de ejercicio físico no competitivo (musculación, aerobic, etc.), de los cuales 402 eran hombres y 325 mujeres, con edades comprendidas entre los 16 y 78 años $(M=32.57$, DT $=11.39)$, de diferentes centros deportivos en una ciudad española. 
Álvaro Sicilia C., Cornelio Águila S., David González-Cutre, Juan Antonio Moreno-M.

\section{Instrumentos}

Cuestionario de Medida de las Estrategias

Motivacionales

Para evaluar la percepción del clima motivacional de los practicantes se utilizó el Cuestionario de Medida de las Estrategias Motivacionales (Cervelló, Moreno, Del Villar \& Reina, 2007). Este cuestionario estaba encabezado por el enunciado "En el centro deportivo..." y compuesto por un total de 24 ítems: 12 ítems para el factor climatarea (e.g. "nos animan a que nos ayudemos entre compañeros durante las tareas") y 12 ítems para el factor clima-ego (e.g. "sólo se evalúa el resultado final, sin importar si progreso respecto a cómo lo hacía antes"). Dicho cuestionario utilizaba una escala Likert de 1 (totalmente en desacuerdo) a 7 (totalmente de acuerdo). En este estudio se obtuvo un valor alfa de Cronbach de 0.77 para el climatarea y 0.78 para el clima-ego.

Cuestionario de las Concepciones de la

Naturaleza de la Habilidad Atlética-2 (CNAAQ-2)

Para medir las creencias implícitas de habilidad se empleó la versión española (González-Cutre et al., 2007) del Conceptions of the Nature of Athletic Ability Questionnaire-2 (Biddle et al., 2003). El instrumento se componía de dos factores: creencia incremental (e.g. "necesitas aprender y trabajar duro para ser bueno/a en la actividad física") y creencia de entidad (e.g. "tienes un determinado nivel de habilidad física y no puedes hacer mucho por mejorarlo"). Estaba compuesto por un total de 12 ítems, encabezados por el enunciado "Tus creencias sobre tu habilidad física son...", que se respondían mediante una escala tipo Likert del 1 (totalmente en desacuerdo) al 5 (totalmente de acuerdo). En este estudio se obtuvieron valores alfa de Cronbach de 0.72 para la creencia incremental y 0.70 para la creencia de entidad.

\section{Motivación intrínseca}

Se empleó el factor regulación intrínseca de la versión traducida al castellano por Moreno, Cervelló \& Martínez (2007) del Behavioural Regulation in Exercise Questionnaire-2 (Markland \& Tobin, 2004). Este factor, encabezado por el enunciado "Yo hago ejercicio...", estaba compuesto por cuatro ítems (e.g. "porque creo que el ejercicio es divertido") que se respondían en una escala Likert de 0 (nada verdadero para mí) a 4 (totalmente verdadero para mí). El análisis de fiabilidad reveló un valor alfa de Cronbach de 0.77 .

\section{Experiencia autotélica}

Se utilizó el factor experiencia autotélica de la versión española (González-Cutre, Sicilia, Moreno \& Fernández-Balboa, 2009) de la Dispositional Flow Scale-2 (Jackson \& Eklund, 2002). Este factor medía la propensión del practicante a la experiencia autotélica y constaba de cuatro ítems (e.g. "me gusta lo que experimento con mi ejecución y me gustaría sentirlo de nuevo") que se respondían mediante una escala tipo Likert de 1 (nunca) a 5 (siempre). El encabezado fue "Cuando práctico ejercicio físico...". Dicho factor mostró un valor alfa de Cronbach de 0.80 .

\section{Procedimiento}

Se estableció contacto con los dirigentes de los diferentes centros deportivos para informarles de los objetivos y pedirles su colaboración. La administración de los cuestionarios fue llevada a cabo por el investigador principal, dando las instrucciones necesarias para la cumplimentación e insistiendo en el anonimato y sinceridad en las respuestas. La participación fue voluntaria y se respetaron todos los procedimientos éticos de recogida de datos.

\section{Análisis de datos}

En primer lugar, se calcularon los estadísticos descriptivos y las correlaciones bivariadas de las variables de estudio. Seguidamente, se testó un 
modelo de ecuaciones estructurales en dos pasos (Anderson \& Gerbing, 1988). Como primer paso se testó la validez de constructo del modelo de medida utilizado a través de un análisis en el que las diferentes variables latentes correlacionaban libremente. El segundo paso fue un modelo estructural que analizaba las relaciones predictivas existentes entre los climas motivacionales percibidos, las creencias implícitas de habilidad, la motivación intrínseca, y la experiencia autotélica. Por último, se comprobó la invarianza del modelo por sexo a través de un análisis multigrupo. Los diferentes análisis se llevaron a cabo con los paquetes estadísticos SPSS 15.0 y AMOS 7.0.

\section{Resultados}

\section{Análisis preliminar}

En la Tabla 1 se puede observar que los practicantes reflejaron una mayor puntuación en clima-tarea que en clima-ego y una mayor puntuación en creencia incremental que en creencia de entidad. Además, se obtuvieron puntuaciones moderadamente altas en motivación intrínseca y experiencia autotélica respecto al rango de oscilación de cada escala.

El análisis de correlaciones bivariadas mostró que el clima-tarea se relacionaba positivamente con la creencia incremental, mientras que el clima- ego se relacionaba positivamente con la creencia de entidad. La correlación positiva del clima-tarea con la motivación intrínseca y experiencia autotélica se mostró más fuerte que la correlación negativa del clima-ego con estas dos últimas variables. La creencia incremental se relacionaba positivamente con la motivación intrínseca, mientras que la creencia de entidad se relacionaba negativamente. La experiencia autotélica se relacionaba positivamente con la motivación intrínseca.

\section{Modelo de ecuaciones estructurales}

Se llevó a cabo un modelo de ecuaciones estructurales para testar las relaciones hipotetizadas entre las variables de estudio. Para todas las variables latentes consideradas en el modelo, se parcelaron sus ítems en dos grupos homogéneos cuyas medias se emplearon como indicadores.

Puesto que el coeficiente de Mardia fue elevado (32.79), se utilizó el método de estimación de máxima verosimilitud junto con el procedimiento de bootstrapping. Este procedimiento proporciona una media de las estimaciones obtenidas del remuestreo bootstrap y su error estándar. Además, compara los valores estimados sin el bootstrap con las medias obtenidas con el remuestreo, indicando el nivel de sesgo. Atendiendo a los intervalos de confianza (diferencia entre los valores estimados más altos y más bajos en los diferentes remuestreos)

TABLA 1

Estadísticos descriptivos y correlaciones bivariadas de todas las variables

\begin{tabular}{lcccccccccc}
\hline \multicolumn{1}{c}{ Variables } & M & DT & Asimetría & Curtosis & 1 & 2 & 3 & 4 & 5 & 6 \\
\hline 1. Clima tarea & 4.81 & 0.85 & -0.27 & 0.49 & - & $-0.27^{* *}$ & $0.32^{* *}$ & $-0.11^{* *}$ & $0.27^{* *}$ & $0.39^{* *}$ \\
2. Clima ego & 2.60 & 0.87 & 0.28 & -0.28 & - & - & $-0.13^{* *}$ & $0.36^{* *}$ & $-0.15^{* *}$ & $-0.17^{* *}$ \\
3. Creencia incremental & 4.25 & 0.60 & -1.02 & 1.18 & - & - & - & 0.03 & $0.26^{* *}$ & $0.29 * *$ \\
4. Creencia entidad & 2.02 & 0.72 & .53 & -0.26 & - & - & - & - & $-0.08^{*}$ & $-0.14^{* *}$ \\
5. Motivación intrínseca & 3.15 & 0.76 & -.98 & 0.90 & - & - & - & - & - & $0.52^{* *}$ \\
6. Experiencia autotélica & 4.25 & 0.61 & -0.76 & 0.89 & - & - & - & - & - & - \\
\hline
\end{tabular}

$* p<0.05 ; * p<0.01$

Fuente: elaboración propia. 
de los pesos de regresión y los pesos de regresión estandarizados, se podía apreciar que el cero no estaba dentro de los límites de confianza, lo que indicaba que los valores estimados eran significativamente distintos de cero. Esto permitía asumir que los resultados de las estimaciones eran robustos y, por tanto, no se veían afectados por la falta de normalidad (Byrne, 2001).

Se utilizó la matriz de covarianza como entrada para el análisis de datos y se emplearon diferentes índices para comprobar la bondad de ajuste del modelo. Se analizó el coeficiente $\chi^{2}$, la ratio entre chi cuadrado y los grados de libertad $\left(\chi^{2} / g l\right)$, el CFI (Comparative Fit Index), el IFI (Incremental Fit Index), el TLI (Tucker Lewis Index), el RMSEA (Root Mean Square Error of Approximation) y el SRMR (Standardized Root Mean Square Residual). Puesto que el $\chi^{2}$ es muy sensible al tamaño muestral y con muestras elevadas la $p$ tiende a ser significativa (Jöreskog \& Sörbom, 1993), resulta conveniente tener en cuenta el coeficiente $\chi^{2} /$ $g l$, que se puede considerar aceptable cuando es inferior a 5 (Bentler, 1989). Según Hu y Bentler (1999), valores de CFI, IFI y TLI superiores a .95, y valores de .06 o inferiores para el RMSEA y .08 para el SRMR, indican un buen ajuste del modelo.

Para dar validez de constructo al modelo de medida utilizado se llevó a cabo un análisis en el que las diferentes variables latentes correlacionaban libremente. Los índices obtenidos fueron: $\chi^{2}$ $(39, N=727)=151.21, p=.00 ; \chi^{2} / g l=3.88 ;$ $\mathrm{CFI}=0.95 ; \mathrm{IFI}=0.95 ; \mathrm{TLI}=0.92 ; \mathrm{RMSEA}=$ 0.06; SRMR $=0.04$. Las correlaciones entre las variables latentes oscilaron entre -0.39 y 0.67 . Estos resultados indicaban que el modelo de medida era aceptable.

Inicialmente, se testó un modelo de ecuaciones hipotetizado donde se planteaba que el clima-tarea predeciría positivamente la creencia incremental, mientras que el clima-ego predeciría positivamente la creencia de entidad. La creencia incremental predeciría positivamente la motivación intrínseca, mientras que la creencia de entidad la predeciría negativamente. La motivación intrínseca predeciría positivamente la experiencia autotélica. Este modelo obtuvo los siguientes índices de ajuste: $\chi^{2}$
$(48, N=727)=226.66, p=.00 ; \chi^{2} / g l=4.72 ;$ $\mathrm{CFI}=0.92 ; \mathrm{IFI}=0.92 ; \mathrm{TLI}=0.90 ; \mathrm{RMSEA}=$ $0.07 ; \mathrm{SRMR}=0.06$

Aunque los índices de ajuste del modelo hipotetizado fueron aceptables, sin embargo, los índices de modificación reflejaron que el modelo mejoraba sustancialmente si se introducía un parámetro entre el clima-tarea y la motivación intrínseca y el clima-tarea y la experiencia autotélica. Esto indicaba que la relación entre clima-tarea y motivación intrínseca y entre clima-tarea y experiencia autotélica, se podía dar de forma directa, además de mediada por otras variables. Los resultados del modelo con esta modificación (Figura 1) mostraron los siguientes índices de ajuste: $\chi^{2}(46, N=727)$ $=161.07, p=.00 ; \chi^{2} / g l=3.50 ; \mathrm{CFI}=0.95 ; \mathrm{IFI}$ $=0.95 ;$ TLI $=0.93$; RMSEA $=0.06$; $\mathrm{SRMR}=$ 0.04 . Todas las relaciones fueron significativas, oscilando los pesos de regresión estandarizados entre -0.14 y 0.64 , con una varianza total explicada de la experiencia autotélica del $55 \%$.

\section{Análisis de la invarianza factorial}

Para comprobar si el modelo estructural era invariante por sexo, se realizó un análisis multigrupo. En la Tabla 2 se pueden observar los índices de ajuste para los seis modelos comparados. No se encontraron diferencias significativas entre el modelo sin restricciones (Modelo 1) y los modelos con invarianza en los pesos de medida (Modelo 2), en los pesos estructurales (Modelo 3), en las covarianzas estructurales (Modelo 4) y en los residuos estructurales (Modelo 5). Estos resultados dan apoyo a la existencia de invarianza del modelo por sexo.

\section{Discusión}

Dada la escasez de estudios sobre los factores que favorecen la experiencia autotélica en el ejercicio físico recreativo, el objetivo de este trabajo fue analizar la influencia del clima motivacional percibido, la creencia de habilidad de los participantes y la motivación intrínseca sobre la experiencia autotélica en un entorno de práctica físicodeportiva de ocio. Asumiendo la potencial influencia de 


\section{FIGURA 1}

Modelo de ecuaciones estructurales mostrando las relaciones entre los climas motivacionales, las creencias de habilidad, la motivación intrínseca y la experiencia autotélica

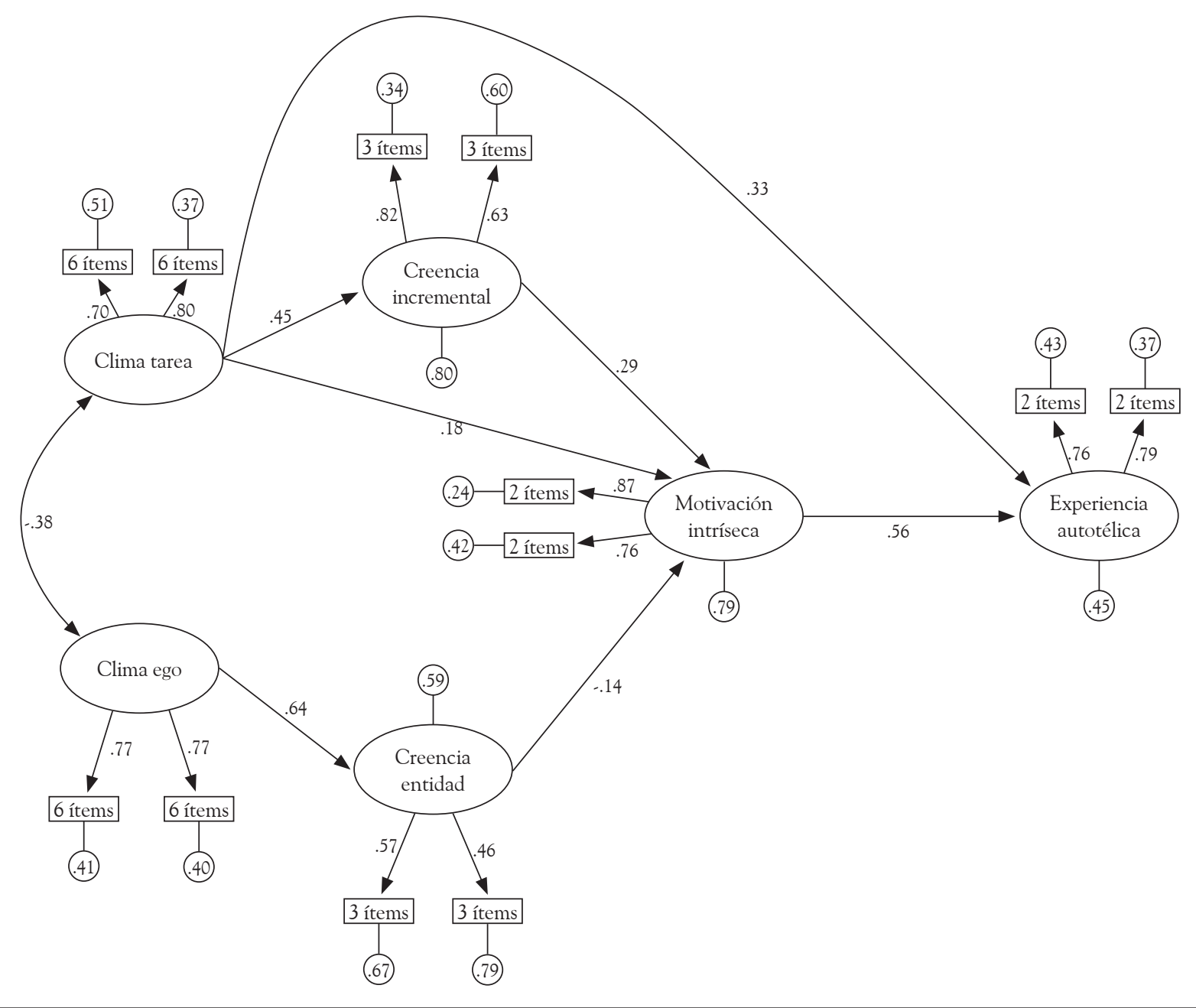

Nota. Todos los parámetros están estandarizados y son estadísticamente significativos. Las varianzas residuales se muestran en los pequeños círculos.

Fuente: elaboración propia.

estos factores, se testó un modelo explicativo de las relaciones predictivas entre dichas variables.

Los resultados del modelo de ecuaciones estructurales revelaron que el clima-tarea predecía positivamente la creencia incremental de habilidad, mientras que el clima-ego predecía positivamente la creencia de entidad. Estos resultados van en la línea de los encontrados por Ommundsen (2001) en clases de educación física y suponen una primera aproximación al análisis de la influencia de los climas motivacionales sobre las creencias de habilidad en el ejercicio físico. Los resultados sugieren la posible influencia del monitor deportivo en el desarrollo de las creencias de habilidad de los practicantes. En este sentido, si el monitor da importancia al esfuerzo y la superación personal, el practicante puede llegar a creer que puede mejorar su habilidad, mientras que si el monitor se centra en aspectos de comparación social, el practicante 
Álvaro Sicilia C., Cornelio Águila S., David GonzÁlez-Cutre, Juan Antonio Moreno-M.

TABLA 2

Análisis multigrupo de invarianza del modelo por sexo

\begin{tabular}{ccccccccccc}
\hline Modelos & $\chi^{2}$ & $g l$ & $\chi^{2} / g l$ & $\Delta \chi^{2}$ & $\Delta g l$ & CFI & IFI & TLI & RMSEA & SRMR \\
\hline Modelo 1 & 218.85 & 92 & 2.37 & - & - & 0.95 & 0.95 & 0.92 & 0.04 & 0.04 \\
Modelo 2 & 225.07 & 98 & 2.29 & 6.22 & 6 & 0.95 & 0.95 & 0.93 & 0.04 & 0.04 \\
Modelo 3 & 239.22 & 105 & 2.27 & 20.36 & 13 & 0.94 & 0.94 & 0.93 & 0.04 & 0.04 \\
Modelo 4 & 239.70 & 108 & 2.22 & 20.85 & 16 & 0.94 & 0.94 & 0.93 & 0.04 & 0.04 \\
Modelo 5 & 242.86 & 112 & 2.16 & 24.00 & 20 & 0.94 & 0.94 & 0.93 & 0.04 & 0.04 \\
Modelo 6 & 275.80 & 124 & 2.22 & $56.94 *$ & 32 & 0.94 & 0.94 & 0.93 & 0.04 & 0.04 \\
\hline
\end{tabular}

Nota. Modelo 1 = sin restricciones; Modelo 2 = pesos de medida invariantes; Modelo $3=$ pesos estructurales invariantes; Modelo 4 = covarianzas estructurales invariantes; Modelo $5=$ residuos estructurales invariantes; Modelo $6=$ residuos de medida invariantes.

*p $<0.05$

Fuente: elaboración propia.

tendería a considerar la habilidad como un talento innato y no modificable.

En segundo nivel de predicción el modelo analizado mostró que la creencia incremental de habilidad predijo positivamente la motivación intrínseca, mientras que la creencia de entidad lo hizo de forma negativa. Tal como apuntaron otros estudios en el ámbito de la actividad física y el deporte (Li et al., 2005; Wang \& Biddle, 2001; Wang, Chatzisarantis, Spray \& Biddle, 2002), si un practicante de ejercicio físico concibe su habilidad como algo mejorable es más probable que disfrute con lo que está haciendo que si cree que su habilidad es fija.

En el último nivel de predicción, los datos reflejaron que la motivación intrínseca predijo positivamente la propensión a la experiencia autotélica. Trabajos previos ya indicaron que este tipo de motivación favorecía la aparición del estado de flow en el deporte (Jackson et al., 1998; Kowal \& Fortier, 1999, 2000). No obstante, hasta la fecha no existían trabajos que hubieran analizado la influencia de la motivación intrínseca sobre la experiencia autotélica en la actividad física recreativa. Este estudio sugiere que el hecho de practicar por el placer de la propia actividad podría favorecer la vivencia de experiencias reconfortantes y satisfactorias con mayor frecuencia.
Los resultados del modelo de ecuaciones estructurales también mostraron que la relación entre el clima motivacional y la motivación intrínseca, y entre el clima motivacional y la propensión a la experiencia autotélica, se daban, además de una forma mediada, de forma directa. Estos resultados revelan la importancia que pueden tener los factores ambientales (en este caso el monitor deportivo) en el desarrollo de una motivación positiva y el fomento de experiencias gratificantes para el practicante. Tal hallazgo pone de manifiesto el tipo de estrategias motivacionales que debería utilizar el monitor deportivo, asumiendo que uno de los principales objetivos de los programas de ejercicio físico, en el ámbito del ocio, es que los participantes mantengan la práctica a lo largo de su vida. Para ello, resulta fundamental conseguir una alta propensión a percibir experiencias autotélicas, dado que el disfrute y el placer parecen favorecer el mantenimiento de pautas de ejercicio (Jackson, 1996; Heyward, 2002; Kimiecik, 2000). En este sentido, el fomento del esfuerzo, la mejora y superación personal parecen aspectos clave que se deben tener en cuenta. Los resultados del análisis multigrupo muestran, además, que el modelo explicativo testado es invariante por sexo, lo que acentúa su utilidad para la orientación de programas de actividad física en centros deportivos. 
En conclusión, la principal aportación de este trabajo ha sido el análisis de la relación entre climas, creencias de habilidad, motivación y experiencia autotélica en entornos de actividad física recreativa. Proporcionar entornos que favorezcan un clima orientado a la tarea, puede hacer ver a los participantes sus progresos personales y asumir una creencia modificable de su habilidad, que les permitirá afrontar los ejercicios motivados intrínsecamente y obtener experiencias gratas y divertidas. El clima-tarea se ha mostrado como la variable más importante que es necesario tener en cuenta en el desarrollo de este tipo de experiencias. No obstante, la naturaleza correlacional del estudio impide establecer relaciones causales. Por tanto, se necesitan investigaciones de carácter experimental, donde se comprueben las hipótesis aquí establecidas, así como estudios longitudinales que constaten la eficacia de dichos programas en la adherencia hacia la actividad físicodeportiva.

\section{Referencias}

American College of Sport Medicine. (1995). Guidelines for exercise testing and prescription. Philadelphia: Williams \& Wilkins.

Anderson, J. C. \& Gerbing, D. W. (1988). Structural equation modeling in practice: $\mathrm{A}$ review and recommended two-step approach. Psychological Bulletin, 103, 411-423.

Bentler, P. M. (1989). EQS structural equations program manual. [Statistical Software]. Los Angeles: BMDP.

Biddle, S. J. H., Wang, C. K. J., Chatzisarantis, N. L. D. \& Spray, C. M. (2003). Motivation for physical activity in young people: Entity and incremental beliefs about athletic ability. Journal of Sports Sciences, 21(12), 973-989.

Byrne, B. M. (2001). Structural equation modeling with Amos: Basic concepts, applications, and programming. Mahwah, NJ: Erlbaum.

Cervelló, E., Moreno, J. A., Del Villar, F. \& Reina, R. (2007). Desarrollo y validación de un instrumento de medida de las estrategias motivacionales empleadas en las clases de educación física. Revista
Iberoamericana de Psicología del Ejercicio y el Deporte, 2(2), 53-72.

Csikszentmihalyi, M. (1990). Flow: The psychology of optimal experience. New York: Harper \& Row.

Csikszentmihalyi, M. (1997). Flow: The psychology of happines. London: Rider.

Deci, E. L. \& Ryan, R. M. (1985). Intrinsic motivation and self-determination in human behavior. New York: Plenum.

Deci, E. L. \& Ryan, R. M. (2000). The "what" and "why" of goal pursuits: Human needs and the selfdetermination of behaviour. Psychological Inquiry, 11(4), 227-268.

González-Cutre, D., Martínez Galindo, C., Alonso, N., Cervelló, E., Conte, L. \& Moreno, J. A. (2007). Las creencias implícitas de habilidad y los mediadores psicológicos como variables predictoras de la motivación autodeterminada en deportistas adolescentes. En J. Castellano \& O. Usabiaga (Eds.), Investigación en la Actividad Física y el Deporte II (pp. 407-417). Vitoria: Universidad del País Vasco.

González-Cutre, D., Sicilia, A., Moreno, J. A. \& Fernández-Balboa, J. M. (2009). Dispositional flow in physical education: Relationships with motivational climate, social goals, and perceived competence. Journal of Teading in Phisical Education, 28(4), 422-440.

Heyward, V. H. (2002). Advanced fitness assessment and exercise prescription. Champaign, IL: Human Kinetics.

Hu, L. \& Bentler, P. M. (1999). Cutoff criteria for fit indexes in covariance structure analysis: Conventional criteria versus new alternatives. Structural Equation Modeling, 6(1), 1-55.

Jackson, S. A. (1996). Toward a conceptual understanding of the flow experience in elite athletes. Research Quarterly for Exercise and Sport, 67(1), 76-90.

Jackson, S. A. \& Csikszentmihalyi, M. (1999). Flow in sports. Champaign, IL: Human Kinetics.

Jackson, S. A. \& Eklund, R. (2002). Assessing flow in physical activity: The Flow State Scale-2 and Dispositional Flow Scale-2. Journal of Sport and Exercise Psychology, 24(2), 133-150.

Jackson, S. A., Kimiecik, J. C., Ford, S. \& Marsh, H. W. (1998). Psychological correlates of flow in sport. 
Journal of Sport and Exercise Psychology, 20 (4), 358-378.

Jackson, S. A. \& Marsh, H. W. (1996). Development and validation of a scale to measure optimal experience: The flow state scale. Journal of Sport and Exercise Psychology, 18(1), 17-35.

Jöreskog, K. G. \& Sörbom, D. (1993). LISREL 8: Structural equation modeling with the SIMPLIS command language. Chicago: Scientific Software International.

Kimiecik, J. C. (2000). Learn to love exercise. Psychology Today, 33, 20-22.

Kowal, J. \& Fortier, M. S. (1999). Motivational determinants of flow: Contributions from self-determination theory. Journal of Social Psychology, 139, 355-368.

Kowal, J. \& Fortier, M. S. (2000). Testing relationships from the hierarchical model of intrinsic and extrinsic motivation using flow as a motivational consequence. Research Quarterly for Exercise and Sport, 71(2), 171-181.

Li, W., Lee, A. M. \& Solmon, M. A. (2005). Relationships among dispositional ability conceptions, intrinsic motivation, perceived competence, experience, persistence, and performance. Journal of Teaching in Physical Education, 24(1), 51-65.

Markland, D. \& Tobin, V. (2004). A modification to Behavioural Regulation in Exercise Questionnaire to include an assessment of amotivation. Journal of Sport and Exercise Psychology, 26(2), 191-196.

Moreno, J. A., Cervelló, E. \& Martínez Camacho, A. (2007). Measuring self-determination motivation in a physical fitness setting: validation of the Behavioral Regulation in Exercise Questionnaire-2 (BREQ-2) in a Spanish sample. Journal of Sports Medicine and Physical Fitness, 47(3), 366-378.

Moreno, J. A., López de San Román, M., Martínez Galindo, C., Alonso, N. \& González-Cutre, D. (2008). Peers' influence on exercise enjoyment: A self-determination theory approach. Journal of Sports Science and Medicine, 7, 23-31.

Mutrie, N. \& Woods, C. (2003). How can we get people to become more active? A problem waiting to be solved. En J. McKenna \& C. Riddoch (Eds.),
Perspectives on Health an Exercise (pp. 131-152). London: Palgrave Macmillan.

Nicholls, J. G. (1989). The competitive ethos and democratic educacion. Cambridge, MASS: Harvard University Press.

Nicholls, J. G. (1992). The general and the specific in the development and expression of achievement motivation. En G. C. Roberts (Ed.), Motivation in sport and exercise (pp. 57-91). Champaign, IL: Human Kinetics.

Ntoumanis, N. \& Biddle, S. J. H. (1999). A review of motivational climate in physical activity. Journal of Sport Sciences, 17 (8), 643-665.

Ommundsen, Y. (2001). Students' implicit theories of ability in physical education classes: The influence of the motivational aspects of the learning environment. Learning Environments Research, 4(2), 139-158.

Ryan, R. M., Frederick, C. M., Lepes, D., Rubio, N. \& Sheldon, K. M. (1997). Intrinsic motivation and exercise adherence. International Journal of Sport Psychology, 28, 335-354.

Sarrazin, P., Vallerand, R., Guillet, E., Pelletier, L. \& Cury, F. (2002). Motivation and dropout in female handballers: A 21-month prospective study. European Journal of Social Psychology, 32(3), 395-418.

Stein, G. L., Kimiecik, J. C., Daniels, F. \& Jackson, S. A. (1995). Psychological antecedents of flow in recreational sport. Personality and Social Psychology Bulletin, 21(2), 125-135.

Vallerand, R. J. (1997). Toward a hierarchical model of intrinsic and extrinsic motivation. En M. P.Zanna (Ed.), Advances in experimental social psychology (pp. 271-360). New York: Academic Press.

Vallerand, R. J. (2001). A hierarchical model of intrinsic and extrinsic motivation in sport and exercise. En G. C. Roberts (Ed.), Advances in motivation in sport and exercise (pp. 263-319). Champaign, IL: Human Kinetics.

Vallerand, R. J. (2007). Intrinsic and extrinsic motivation in sport and physical activity. A review and a look at the future. En G. Tenenbaum \& R. C. Eklund (Eds.), Handbook of Sport Psychology (3aㅡ ed., pp. 59-83). New York: John Wiley. 
Wang, C. K. J. \& Biddle, S. J. H. (2001). Young people's motivational profiles in physical activity: A cluster analysis. Journal of Sport and Exercise Psychology, 23(1), 1-22.

Wang, C. K. J. \& Biddle, S. J. H. (2003). Intrinsic motivation towards sports in Singaporean students: The role of sport ability beliefs. Journal of Health Psychology, 8(5), 515-523.
Wang, C. K. J., Chatzisarantis, N. L. D., Spray, C. M. \& Biddle, S. J. H. (2002). Achievement goal profiles in school physical education: Differences in selfdetermination, sport ability beliefs, and physical activity. British Journal of Educational Psychology, 72(3), 433-445. 
